\title{
Evaluation of Astigmatism-Correcting Efficiency and Rotational Stability after Cataract Surgery with a Double-Loop Haptic Toric Intraocular Lens: A 1-Year Follow-Up
}

\author{
Ladislav Viktor Nováček ${ }^{a, d}$ Marie Němcováa Kateřina Tyx ${ }^{a}$

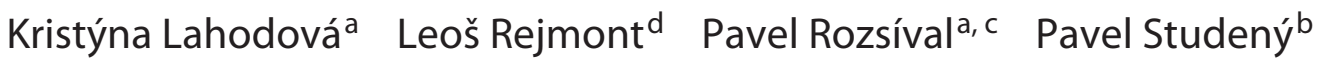 \\ ${ }^{a}$ Department of Ophthalmology, Institute of Aviation Medicine Prague, Prague, Czech Republic; ${ }^{b}$ Department of \\ Ophthalmology Královské Vinohrady University Hospital, and 3rd Faculty of Medicine, Charles University, Prague, \\ Czech Republic; ' Department of Ophthalmology Charles University Prague, Faculty of Medicine in Hradec Králové, \\ Prague, Czech Republic; ${ }^{\mathrm{d} D e p a r t m e n t}$ of Ophthalmology, 1st Faculty of Medicine, Charles University and the \\ Military University Hospital Prague, Prague, Czech Republic
}

\section{Keywords}

Intraocular lens · Astigmatism correction · Toric lens ·

Cataract $\cdot$ Rotational stability

\begin{abstract}
Objectives: The aim of this study was to assess the clinical outcomes, predictability of results, efficiency of astigmatism correction, and rotational stability of the Bi-Flex 677TAY (Medicontur Medical Engineering Ltd., Zsámbék, Hungary) monofocal toric intraocular lens (IOL) designed for cataract patients with astigmatism. Methods: The IOLs were implanted either mono- or binocularly, following routine cataract surgery. Visual and refractive outcomes, as well as off-axis rotation were assessed throughout a 1-year follow-up period. All clinical data for this work were collected retrospectively. Vector analysis based on the Alpins method was performed to assess the efficiency of astigmatism correction. Results: No complications or adverse events occurred during surgery or the follow-up period. IOL implantation brought $88 \%$ of eyes into the $\pm 0.50 \mathrm{D}$, and $100 \%$ into the $\pm 1.00 \mathrm{D}$ range compared to the target spherical equivalent refraction, emmetropia. Astigmatism correction brought
\end{abstract}

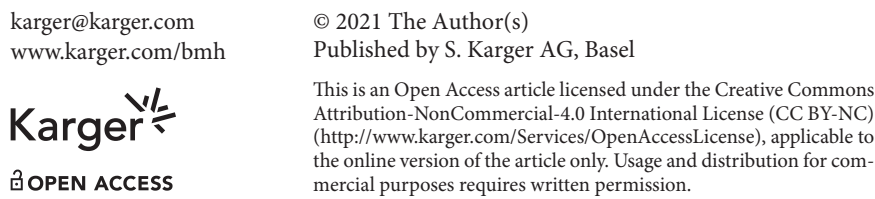

karger@karger.com www.karger.com/bmh

Karger $\stackrel{\text { ' }}{5}$

BOPEN ACCESS

(C) 2021 The Author(s)

Published by S. Karger AG, Basel

This is an Open Access article licensed under the Creative Commons Attribution-NonCommercial-4.0 International License (CC BY-NC) (http://www.karger.com/Services/OpenAccessLicense), applicable to the online version of the article only. Usage and distribution for commercial purposes requires written permission.

similar results: $94 \%$ of eyes had a residual cylindrical error of not higher than $\pm 0.50 \mathrm{D}$, and $97 \%$ were within $\pm 1.00 \mathrm{D}$. Vector analysis resulted in a correction index of 0.96 and a difference vector of 0.17 . Both refractive and visual outcomes showed long-term stability. During the 12-month follow-up period, no eyes had a rotation of $>5^{\circ}$. Absolute rotation after 1 year was $1.42 \pm 1.89^{\circ}\left(\right.$ median $\left.=0^{\circ}\right)$, while signed rotation was $1.06 \pm 2.12^{\circ}$ (median $\left.=0^{\circ}\right)$. Conclusion: The Bi-Flex 677TAY monofocal toric IOL, designed by Medicontur Medical Engineering Ltd., represents an efficient and safe solution for cataract patients with astigmatism. Clinical and refractive outcomes are predictable, and rotational stability ensures long-term visual comfort.

(c) 2021 The Author(s)

Published by S. Karger AG, Basel

\section{Introduction}

In recent years, cataract surgery has evolved from the simple removal and replacement of the opaque crystalline lens to high-precision refractive surgery to avoid further vision correction [1]. Ferrer-Blasco et al. [2], in their 
study that included 4,540 eyes, reported that a remarkable $87 \%$ of cataract patients had preoperative astigmatism. In a recent study of 13,012 eyes (i.e., of 6,506 cataract patients), it was reported that $43.5 \%$ of the eyes had a corneal astigmatism of at least 1.00 diopters (D), which has been shown to have an adverse effect on the best achievable surgical outcome, and is enough to significantly reduce both visual acuity and the quality of postoperative vision when left untreated [3].

In recent years, several toric intraocular lenses (IOLs) have been introduced onto the market that are all designed to efficiently correct preoperative astigmatism. An effective toric IOL is characterized by a high level of astigmatism-correcting ability (meaning that the refractive outcomes are as close as possible to the intended postoperative spherical and cylindrical refraction), high predictability of the refractive and visual outcomes, and excellent rotational stability which prevents the loss of astigmatism correction as a result of off-axis rotation of the lens.

Apart from the latest IOL models designed with high precision, numerous state-of-the-art measurement devices, and the latest advanced calculation formulas that take the results of several biometrical parameters into consideration, sometimes even integrating (or modeling) the astigmatism of the posterior corneal surface, are available $[4,5]$. Precise IOL alignment is a further component contributing to the eventual success rate of surgical astigmatism correction [6].

Based on a literature search for the clinical outcomes following the implantation of the toric IOLs, we realized that the majority of papers published results after a follow-up period of $\leq 6$ months [7-13]. The stability of the refractive and visual results over longer periods of time and of the IOL position are also very important, however. Only a small number of studies have been published on the Bi-Flex 677TA(Y) monofocal, toric, capsular-bag IOL $[14,15]$. The purpose of this investigation was to assess the long-term efficacy and stability of the abovementioned toric lens. Our retrospective evaluation was complemented with a vector analysis based on the Alpins method; it is expected that this will provide a more accurate assessment of the astigmatism-correcting ability of the IOL in question.

\section{Materials and Methods}

\section{Patient Population}

This retrospective, noncomparative, one-site, one-surgeon, one-lens, clinical investigation involved 35 eyes (of 22 cataract patients), all of which had preoperative corneal astigmatism repre- senting an indication for the implantation of an astigmatism-correcting IOL following removal of the cataractous crystalline lens. The pre- and postoperative records of patients receiving mono- or binocular surgery performed by the same experienced surgeon (L.V.N.) between April 2017 and February 2019 were collected and evaluated. The data management and the clinical study adhered to the principles of the Declaration of Helsinki [16].

The subset of patients included in the assessments was selected carefully. Subjects with congenital eye disease, previous corneal laser refractive surgery, amblyopia, uveitis, eye trauma, and/or other corneal or intraocular pathologies were not included in the further assessment.

\section{Pre- and Postoperative Assessment}

Prior to surgery, a detailed ocular examination was performed including corneal topography, aberrometry (TRK-2P, Topcon Europe Medical B.V., Capelle aan den Ijssel, The Netherlands), anterior-segment examination (Oculus Pentacam 70700, OCULUS Optikgeräte $\mathrm{GmbH}$, Wetzlar, Germany), posterior-segment examination with a slit lamp (SL-D4, Topcon Europe Medical B.V.), and optical biometrics including axial length (AXL), anteriorchamber depth (ACD), keratometry (K1, K2), and white-to-white measurements (IOL Master 700, Carl Zeiss Meditec AG, Jena, Germany). Intraocular pressure was also measured in all cases. Monocular distance visual acuity (both uncorrected [UDVA] and corrected [CDVA]) was assessed by using the SC-1600 Snellen Chart optotypes (Nidek Co., Ltd., Tokyo, Japan), while corrected near vision (CNVA) was measured with the Jaeger reading card.

The same measurements were repeated with the same protocols at 1 day, 1 week, 1,2 , and 3 months, and then 1 year postoperatively. The surgical position of the implanted toric IOL was determined and documented by slit-lamp examination during surgery and at each postoperative visit, with measurement performed in artificial mydriasis (slit lamp SL 120 with SL imaging module, Carl Zeiss Meditec AG).

All pre- and postoperative measurements were performed by the same person (M.N.).

Rotational stability was evaluated by comparing the position of the IOL at each postoperative visit to the intended torus position (surgical position). The mean absolute rotation (focusing only on the degree of rotation) and signed rotation (rotation clockwise was counted as a negative and counter-clockwise as a positive rotation) were also determined. Rotation between the intended torus position and the first postoperative visit on day 1 was regarded as misalignment, and between day 1 and the following visits as toric IOL rotation.

The efficiency of astigmatism correction was evaluated with the VectrAK vector analysis software (ASSORT Pty. Ltd., Cheltenham, VIC, Australia) based on the Alpins method $[17,18]$. The analysis is described in detail in the "Vector Analysis" section.

\section{Bi-Flex 677TA $(Y)$}

The Bi-Flex 677TA(Y) monofocal, toric IOL (Medicontur Medical Engineering Ltd., Zsámbék, Hungary) has an overall diameter of $13.0 \mathrm{~mm}$ and an optic diameter of $6.0 \mathrm{~mm}$. The optic is connected to the haptics without haptic angulation (Fig. 1). The double-loop haptic design ensures a large contact angle $\left(2 \times 88.8^{\circ}\right)$ with the wall of the capsular bag [19]. The single-piece IOL is manufactured from a hydrophilic acrylic copolymer with a final water content of $25 \%$ and a refractive index of 1.46 measured at $23^{\circ} \mathrm{C}$ 


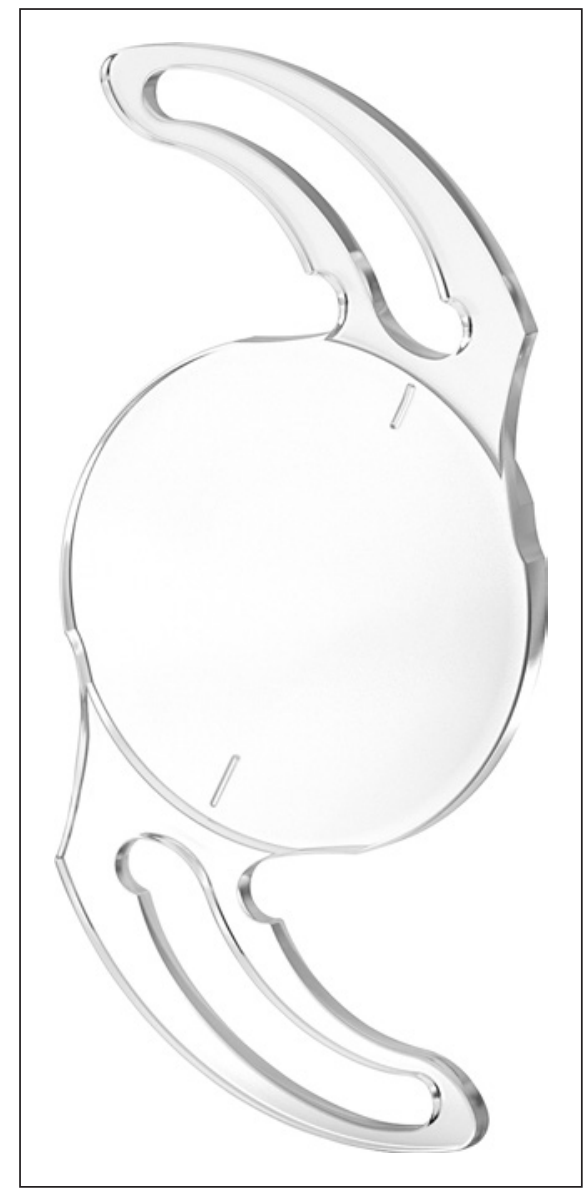

Fig. 1. The Bi-Flex 677 monofocal toric IOL is available as transparent (677TA) and with a blue-light filter (677TAY).

(Abbe No. 58). All lenses include a UV-absorber, while a blue-light filter is optional and depends on the model chosen (677TA represents the transparent model and 677TAY represents the yellow model). The IOL is aspheric with neutral asphericity and has a patented special $360^{\circ}$ square edge to inhibit the migration of lens epithelial cells and consequently prevent the development of posterior-capsule opacification (PCO). The torus is marked with 2 marks indicating the flat axis on the edge of the optic. The lens is available in cylindrical powers of +1.0 to $+10.0 \mathrm{D}$ (IOLs outside of this range are produced on request).

In each case, spherical equivalent (SEQ), cylindrical power (CYL), and the correct position of the optimal IOL chosen for implantation were determined using the online IOL calculator of the manufacturer [20]. All eyes were targeted to emmetropia, and the surgically induced astigmatism (SIA) of the surgeon was also taken into consideration. Calculations were performed using the Haigis formula [21], while the Abulafia-Koch correction recommended by the manufacturer was not applied [5], as posterior corneal astigmatism had already been taken into account by the IOL Master 700 optical biometer.

\section{Surgical Technique}

Under local anesthesia and following pupil dilation, a 2.2- $\mathrm{mm}$, clear corneal incision was made in each case at the position recommended by the IOL calculator, and the anterior chamber was filled with viscoelastic material (Visco-MC 2\%, Medicontur Ltd.; Eye Gel Plus 2.4\%, Kosmetik Konzept KOKO GmbH, Leichlingen, Germany; or Hydromel 2.5\%, Unimed Pharma spol. sro., Bratislava, Slovakia). Circular curvilinear capsulorhexis was performed, and the opaque crystalline lens was removed by phacoemulsification in all cases. The tested IOLs were folded and implanted with the MedJet B1B or MA 2.2 injector (Medicontur Ltd.). In-the-bag implantation with sutureless wound closure was performed in all cases. After the implantation and the removal of the ophthalmic viscosurgical device, the IOL was rotated to its final position by accurately aligning the toric reference marking of the IOL, according to the markerless toric IOL alignment tool of the Callisto eye system (Carl Zeiss Meditec AG).

Antibiotic and antimicrobial eye drops were administered intraoperatively in each case to reduce the risk of surgical infection, while antibiotic and anti-inflammatory eye drops were administered postoperatively.

In the case of binocular implantation, a 1-week interval was maintained between the 2 surgeries.

\section{Vector Analysis}

Vector analysis and graphical displays were performed using the Alpins method [17, 18], facilitated by the VECTrAK astigmatism analysis program v2.4.6 (ASSORT Pty., Ltd.). Analysis was performed according to the protocol previously described by Bachernegg et al. [15]; briefly, based on manifest refraction and topographic astigmatism, astigmatism to the corneal plane was calculated in each case, and preoperative values were compared to the results measured 12 months postoperatively.

Three fundamental vectors and the relationship among them were examined. These are the target-induced astigmatism vector (TIA), the surgically induced astigmatism vector (SIA), and the difference vector (DV) $[17,18]$.

TIA represents the astigmatic change (by magnitude and axis) the surgery was intended to induce, so the actual measured preoperative corneal topographic astigmatism was used. SIA refers to the astigmatic change (by magnitude and axis) that the surgery actually induced. The SIA is a quantity calculated by subtracting the phacoemulsification-adjusted preoperative corneal astigmatism from the postoperative refractive cylinder calculated to the corneal plane. Based on these 2 vectors, the correction index (CI) can be determined. The CI is calculated by dividing SIA by TIA. The CI value is preferably 1.0. It is $>1.0$ if overcorrection occurs and $<1.0$ if there is undercorrection.

The induced astigmatic change (by magnitude and axis) that enables the initial surgery to achieve its intended target is described by the third fundamental vector, the DV. The DV is the actual measured postoperative refraction remaining after treatment. It is an absolute measure of success and is preferably zero.

The cumulative histogram of the magnitudes of the preoperative corneal astigmatism and postoperative refractive astigmatism at the corneal plane, and the double-angle plots of preoperative and postoperative refractive astigmatism (including centroid values with SDs and 95\% confidence ellipses of the dataset and of the centroid values) were plotted according to the method presented by Abulafia et al. [22]. 


\section{Statistical Analysis}

All data were collected in Microsoft Excel (Microsoft Inc., Redmond, WA, USA), and later evaluated using GraphPad Prism v8.3.0 software (GraphPad Software Inc., San Diego, CA, USA). All pre- and postoperative data collected during the study are available after deidentification from the Mendeley Data depository database (DOI: 10.17632/r2v99nw7vg.2) [23].

Pre- and post-operative data on 22 patients (35 eyes) were included in the analyses. Descriptive statistics (mean, SD, median, minimum, maximum, and 95\% confidence ellipse) were calculated in all cases. All variables were tested for normal distribution using the D'Agostino and Pearson test. Depending on the results, comparisons between matching pre- and postoperative variables were performed using either the paired two-tailed $t$ test (in the case of normal distribution) or the Mann-Whitney $U$ test (when a nonparametric test was required). Visual and refractive outcomes are presented according to the standards for reporting refractive outcomes of IOL-based refractive surgery published recently by Reinstein et al. [24]. The stability of refractive outcomes was also assessed during the follow-up period.

Results are presented as mean \pm SD along with the range defined by the minimum and maximum values in brackets in the case of each analysis. $p \leq 0.05$ were considered to be statistically significant in all cases.

\section{Results}

This retrospective study evaluates the visual and refractive characteristics of 35 eyes of 22 astigmatic cataract patients preoperatively, and then after cataract surgery that included the implantation of the toric Bi-Flex 677TA(Y) IOL. Apart from the assessment of visual improvement and the correction of refractive errors, the rotational stability of the lens and the efficacy of astigmatism correction using vector analysis were also determined.

Table 1 presents the characteristics of the study population. Only subjects diagnosed with normal ocular conditions during biomicroscopy or fundoscopy examinations were included in the analyses.

The mean spherical equivalent of the implanted IOLs was $22.2 \pm 2.52 \mathrm{D}(16.0 \mathrm{D} ; 27.5 \mathrm{D})$, while the mean cylindrical power of the lenses was $1.70 \pm 0.86 \mathrm{D}(1.0 \mathrm{D} ; 3.75$ D).

No IOL-related complications could be detected during surgery in any of the cases. Intraocular pressure (IOP) remained in the normal range $(<20 \mathrm{~mm} \mathrm{Hg})$ during and following the operation $(15.6 \pm 3.05 \mathrm{~mm} \mathrm{Hg}$ preoperatively; $15.7 \pm 2.66 \mathrm{~mm} \mathrm{Hg}$ at day 1 , and $14.6 \pm 2.72 \mathrm{~mm}$ $\mathrm{Hg} 12$ months after surgery). On the first postoperative day, 1 eye showed symptoms of inflammation characterized by anterior-chamber cells (grade $3+$ ) and flare, and 9 eyes had developed slight corneal edema, but these con-

Astigmatism Correction with a Double-

Loop Haptic IOL
Table 1. Demographic and preoperative characteristics of the study population

$\begin{array}{ll}\text { Gender } & \\ \quad \text { Female } & 14(63.6) \\ \quad \text { Male } & 8(36.4) \\ \text { Age, years } & 66.7 \pm 5.4(50-74) \\ \text { AXL, mm } & 23.22 \pm 1.20(21.22-25.92) \\ \text { ACD, mm } & 2.94 \pm 0.49(1.85-4.03) \\ \text { K1, D } & 42.74 \pm 1.93(40.14-47.14) \\ \text { K2, D } & 44.53 \pm 1.91(41.13-48.73) \\ \text { CYL, D } & -1.54 \pm 1.23(-4.50 \text { to }-1.50) \\ \text { SEQ, D } & 0.46 \pm 2.61(-6.75 \text { to }+5.13) \\ \text { UDVA (decimal) } & 0.32 \pm 0.18(0.01-0.60) \\ \text { BDVA (decimal) } & 0.65 \pm 0.19(0.15-1.00) \\ \text { UNVA (Jaeger lines) } & 8.46 \pm 4.38(0.00-13.0) \\ \text { BNVA (Jaeger lines) } & 2.33 \pm 1.76(0.00-10.00) \\ \text { IOP, mm Hg } & 15.7 \pm 3.07(10.0-25.0) \\ \end{array}$

Values are expressed as mean \pm SD (range) or $n(\%)$.

ditions had cleared up by the second postoperative week in all the affected cases. No further abnormalities that could have correlated directly with the surgery or the IOL itself could be revealed during the 12-month follow-up period. During the 3-month postoperative visit, 1 patient was diagnosed with binocular dry-type age-related macular degeneration (AMD), but her visual acuity and refractive values did not reflect her retinal condition. By the end of the first postoperative year, 1 patient implanted monocularly with the toric IOL developed an epiretinal membrane, which resulted in a significant deterioration of her vision; she was thus excluded from the evaluation of the 12 -month results. One other patient moved abroad during the follow-up period and was lost to follow-up. PCO was detected in 9 eyes in the course of the 12-month follow-up.

\section{Visual Outcomes}

A significant improvement was achieved in both monocular UDVA and CDVA compared to the preoperative values (Table 2). Figure 2 demonstrates that all eyes had a Snellen visual acuity of at least 20/25 (Fig. 2a), and that most of the eyes achieved the best possible visual acuity (UDVA was the same or better than CDVA in $90.9 \%$ of the cases; Fig. 2b). The results were shown to be stable over time, with no remarkable differences found between those obtained 3 months and 1 year after surgery (Table 2; Fig. 2c, d).

As a consequence of cylindrical correction, a significant increase in corrected near visual acuity could be also 
Table 2. Visual acuity, required spherical and cylindrical correction, and differences between the pre- and postoperative measurements after the implantation of the Bi-Flex 677TA(Y) astigmatism-correcting IOL

\begin{tabular}{|c|c|c|c|c|c|}
\hline Correction & Preoperative & $\begin{array}{l}\text { Postoperative } \\
\text { (at } 3 \text { months) }\end{array}$ & $\begin{array}{l}\text { Postoperative } \\
\text { (at } 12 \text { months) }\end{array}$ & $\begin{array}{l}p \text { value } \\
\text { preop vs. M3* }\end{array}$ & $\begin{array}{l}p \text { value } \\
\text { M3 vs. M12* }\end{array}$ \\
\hline \multicolumn{6}{|l|}{ Distance } \\
\hline \multicolumn{6}{|l|}{ UDVA, D } \\
\hline Mean \pm SD & $0.32 \pm 0.18$ & $0.92 \pm 0.08$ & $0.93 \pm 0.13$ & \multirow[t]{2}{*}{$<0.0001$} & \multirow[t]{2}{*}{0.0339} \\
\hline Range & $0.01-0.60$ & $0.80-1.00$ & $0.50-1.00$ & & \\
\hline \multicolumn{6}{|l|}{ CDVA, D } \\
\hline Mean \pm SD & $0.65 \pm 0.19$ & $0.97 \pm 0.06$ & $0.96 \pm 0.08$ & \multirow[t]{2}{*}{$<0.0001$} & \multirow[t]{2}{*}{0.9146} \\
\hline Range & $0.15-1.00$ & $0.80-1.00$ & $0.70-1.00$ & & \\
\hline \multicolumn{6}{|l|}{ SPH, D } \\
\hline Mean \pm SD & $0.61 \pm 2.23$ & $-0.10 \pm 0.19$ & $0.01 \pm 0.29$ & \multirow[t]{2}{*}{0.0396} & \multirow[t]{2}{*}{0.0337} \\
\hline Range & -4.0 to +5.00 & -0.50 to +0.25 & -0.75 to +0.75 & & \\
\hline \multicolumn{6}{|l|}{ CYL, D } \\
\hline Mean \pm SD & $-1.02 \pm 1.10$ & $-0.19 \pm 0.29$ & $-0.17 \pm 0.31$ & \multirow[t]{2}{*}{$<0.0001$} & \multirow[t]{2}{*}{0.6563} \\
\hline Range & -3.75 to +1.00 & -1.00 to 0.00 & -1.00 to 0.00 & & \\
\hline \multicolumn{6}{|l|}{ SEQ, D } \\
\hline Mean \pm SD & $0.10 \pm 2.25$ & $-0.19 \pm 0.30$ & $-0.08 \pm 0.41$ & \multirow[t]{2}{*}{0.1739} & \multirow[t]{2}{*}{0.0557} \\
\hline Range & -4.50 to +4.88 & -0.75 to 0.25 & -1.25 to 0.75 & & \\
\hline \multicolumn{6}{|l|}{ Near } \\
\hline \multicolumn{4}{|l|}{ UNVA (Jaeger) } & \multirow[t]{3}{*}{ n.a. } & \\
\hline Mean \pm SD & $8.46 \pm 4.38$ & n.a. & n.a. & & n.a. \\
\hline Range & $0-13$ & & & & \\
\hline \multicolumn{6}{|l|}{ CNVA (Jaeger) } \\
\hline Mean \pm SD & $2.33 \pm 1.76$ & $1.09 \pm 0.38$ & $1.27 \pm 0.72$ & \multirow[t]{2}{*}{$<0.0001$} & \multirow[t]{2}{*}{0.5000} \\
\hline Range & $0-10$ & $1-3$ & $1-4$ & & \\
\hline
\end{tabular}

Bold type denotes significance. ${ }^{*} p$ values of no more than 0.05 were considered statistically significant.

observed ( $p<0.0001$; Table 2). Three months postoperatively, all patients were able to read at least line 2 on the Jaeger reading chart, and the results did not change significantly up to the end of the first postoperative year $(p=0.5000$; Fig. 3a, b).

\section{Refractive Outcome Predictability}

Before the cataract surgery and implantation of the toric IOL, only $31.4 \%$ of eyes had a spherical equivalent refraction of no more than $0.5 \mathrm{D}$, and $42.9 \%$ of eyes were within $1.0 \mathrm{D}$ from emmetropia. Three months postoperatively, $87.9 \%$ of eyes were detected to be within $0.5 \mathrm{D}$, and all (100\%) were within 1.0 D (Fig. 4a). Cylindrical correction was found to be similarly effective. While only $40 \%$ of eyes had a refractive cylinder of no more than $0.5 \mathrm{D}$, and $62.9 \%$ of eyes were within $1.0 \mathrm{D}$ according to the preoperative measurements, surgery brought about a significant increase in their cylindrical correction; $94 \%$ were found to be within $0.5 \mathrm{D}$, and $100 \%$ of the eyes were within the $1.0 \mathrm{D}$ range (Fig. $4 \mathrm{~b}$ ). In the majority of the cases, these refractive outcomes remained stable during the first postoperative year (Fig. 4c, d). The preoperative estimation of refractive outcomes was shown to be highly predictable; the mean error in residual refraction (achieved values vs. preoperative estimations) was $-0.12 \pm 0.33$ $(-0.66$ to 0.72$)$ diopters in the case of SEQ, and $0.18 \pm 0.31$ $(-0.55$ to 0.77$)$ diopters in the case of CYL.

Surgery and the implantation of a toric IOL brought a significant improvement in the manifest spherical and cylindrical refraction of the eyes. Table 2 presents the refractive results measured 3 and 12 months postoperatively; these refractive outcomes had already actually been achieved immediately after implantation of the toric lens. Comparing the residual refraction values (SEQ and CYL) at each postoperative visit, the results proved to be stable over time, with no significant changes observed (Table 2).

\section{Vector Analysis}

The efficiency of the Bi-Flex 677TA(Y) toric lens in correcting corneal astigmatism was examined in detail using vector analysis based on the Alpins method $[17,18]$. Figure 5 shows the cumulative histogram of the magni- 


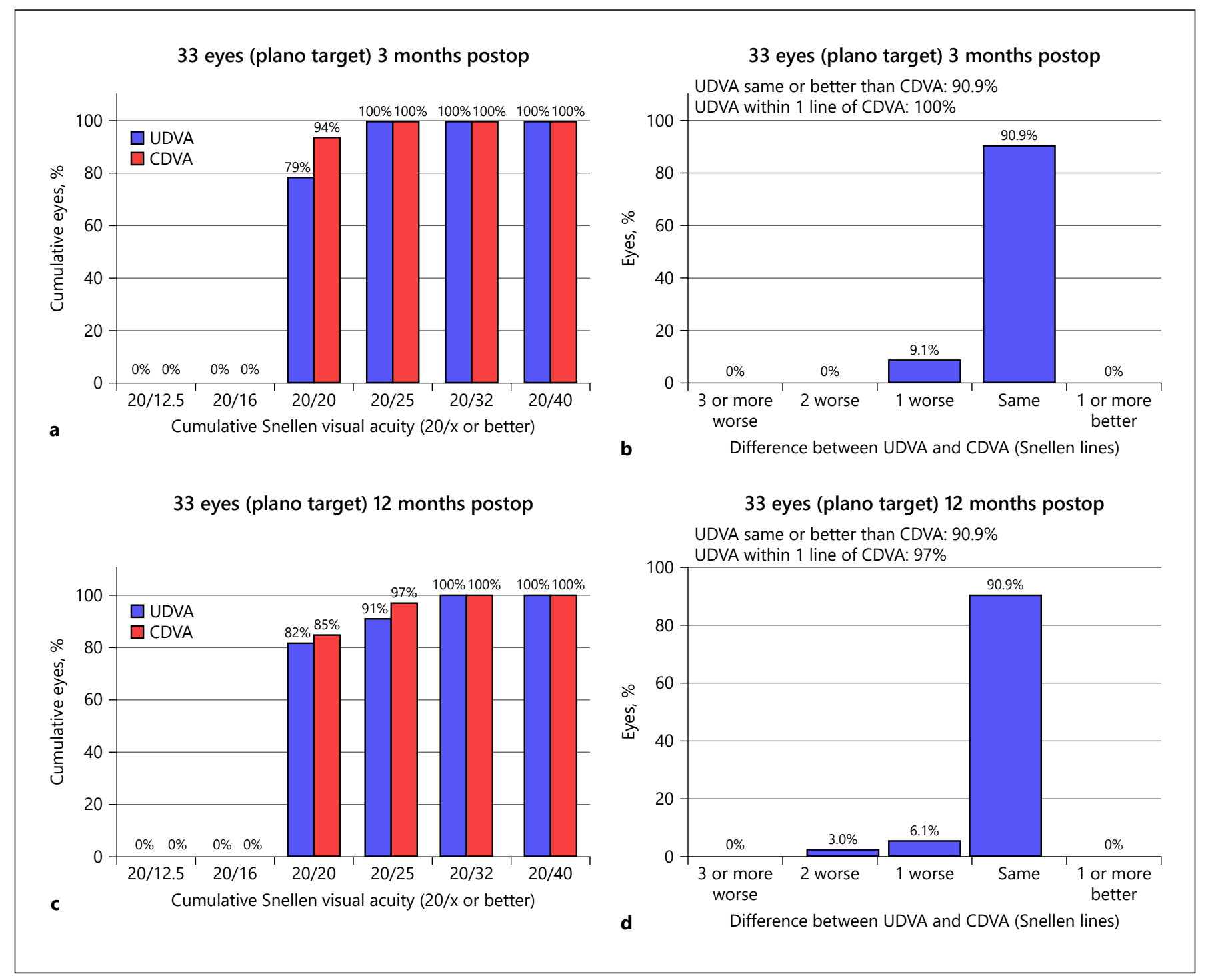

Fig. 2. a, c Cumulative histograms of uncorrected and corrected distance visual acuity measured 3 and 12 months postoperatively. $\mathbf{b}, \mathbf{d}$ Results are similar at both postoperative visits. Uncorrected visual acuity is close to the best possible values.

tudes of preoperative corneal astigmatism and postoperative refractive astigmatism at the corneal plane. The double-angle plots of pre- and postoperative refractive astigmatism (including centroid values with SDs and 95\% confidence ellipses of the dataset and of the centroid values) are plotted in Figure 6. From the preoperative mean absolute corneal astigmatism of $2.04 \pm 1.02 \mathrm{D}$, refractive astigmatism decreased to a mean of $0.17 \pm 0.31 \mathrm{D} 12$ months postoperatively.

Vector analysis could be performed with the pre- and postoperative data of 24 eyes. Postoperative measure- ments were performed 1 year postoperatively. The fundamental vectors of astigmatism (TIA and SIA), and their relationships (DV and CI) are presented in Figure $7 \mathrm{a}-\mathrm{d}$, respectively. TIA had an arithmetic mean of $1.5 \mathrm{D}$, and the vector mean was $1.01 \mathrm{D} \mathrm{Ax} 2^{\circ}$ (Fig. 7a). SIA was similar, having an arithmetic mean of $1.45 \mathrm{D}$ and a vector mean of $0.95 \mathrm{D} \mathrm{Ax} 5^{\circ}$ (Fig. 7b). These similar values resulted in a low DV of $0.17 \mathrm{D}$ (vector mean: $0.09 \mathrm{D} \mathrm{Ax} 158^{\circ}$; Fig. 7c), which is close to the optimal DV value of zero. Reflecting the DV, the CI was shown to be high; the arithmetic mean was 0.96 (Fig. 7d). This is close to the optimal 


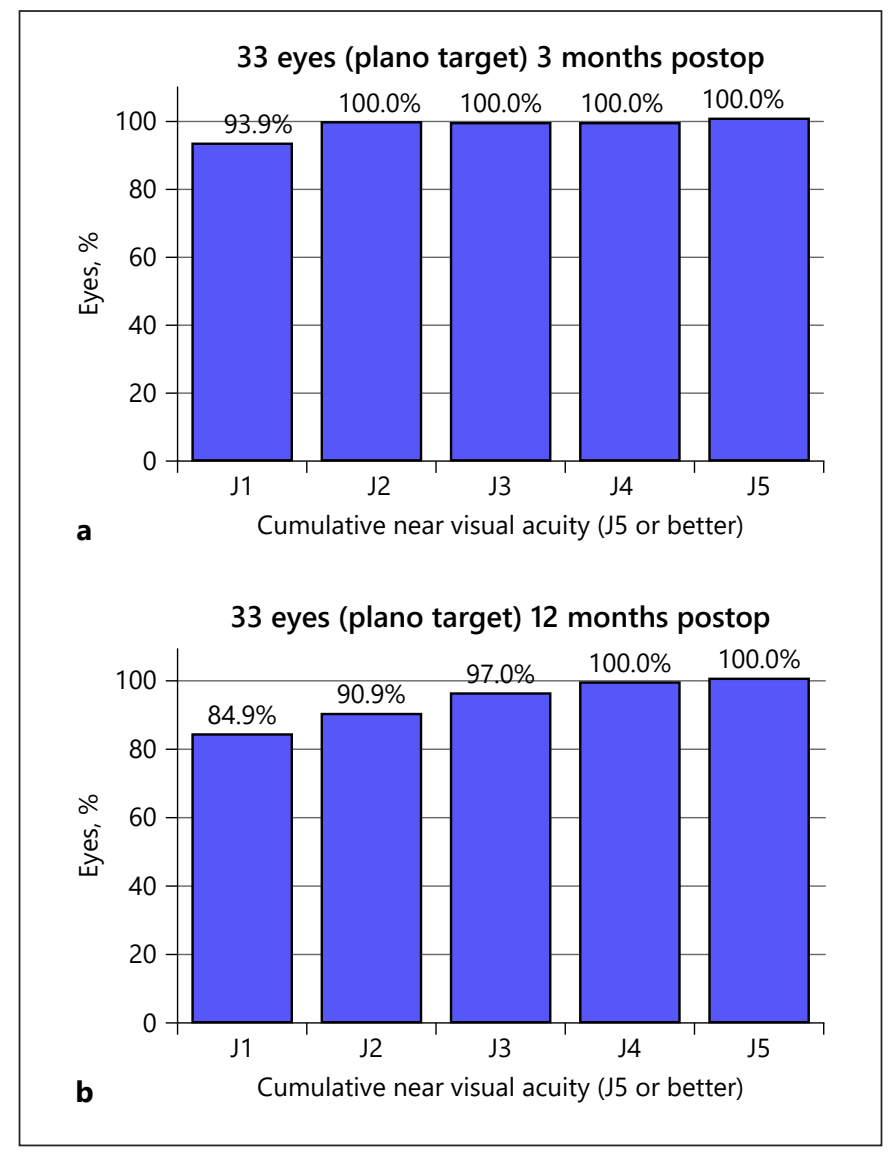

Fig. 3. a, b Cumulative histograms of corrected near visual acuity measured 3 and 12 months postoperatively with the Jaeger reading card are similar $(p=0.500)$.

1.00 value but shows a slight undercorrection of astigmatism.

\section{Rotational Stability of the IOL}

Rotational stability of the lens was examined according to the conventional method, i.e., comparing the position of the IOL at each postoperative visit to the intended (surgical) position of the lens. In the first $24 \mathrm{~h}$ after surgery, only an average rotation of $0.57^{\circ} \pm 2.19^{\circ}$ could be observed, and there was no need for repositioning in any of the cases. Based on this, it can be stated that there was no misalignment in any cases. Further rotations are presented in Table 3. In the case of signed rotation, clockwise rotation was counted as a negative rotation and counterclockwise rotation as a positive rotation. Absolute rotation disregards the direction of rotation, taking only the magnitude of the rotation into consideration. A rotation of $>5^{\circ}$ in any direction could not be observed in any of the
Table 3. Rotational stability compared to the surgical position of the implanted IOL throughout the 12-month follow-up period

\begin{tabular}{lll}
\hline Follow-up visit & Signed & Absolute \\
\hline Day 1 & $0.63 \pm 2.18$ & $1.37 \pm 1.80$ \\
Day 7 & $0.74 \pm 2.63$ & $1.84 \pm 2.00$ \\
Month 1 & $0.54 \pm 2.54$ & $1.63 \pm 1.90$ \\
Month 3 & $0.91 \pm 2.67$ & $1.88 \pm 2.09$ \\
Month 12 & $1.06 \pm 2.12$ & $1.42 \pm 1.89$ \\
\hline
\end{tabular}
$\left({ }^{\circ}\right)$

Results are presented as mean $\pm \mathrm{SD}$, and expressed in degrees

cases, with all IOLs $(100 \%)$ remaining within $5^{\circ}$ relative to the intended IOL position during the entire follow-up period.

\section{Discussion}

The aim of our retrospective data collection was to assess the visual and refractive outcomes, and efficacy of the astigmatism correction as well as evaluate the refraction and rotational stability of the clinical results after the implantation of the Bi-Flex 677TA(Y) monofocal, toric, capsular-bag IOL. Only a limited number of publications are available on this particular lens $[14,15]$, and only 1 presents results obtained during a longer follow-up period (12 months) [15]. Hardly any studies are available that include postoperative monitoring for longer than 3 months, with most publications on astigmatism-correcting IOLs presenting their results after $\leq 3$ months of follow-up. Nevertheless, it is of major importance to increase knowledge on the long-term stability of all (toric) lenses, as rotation is known to reduce the astigmatism-correcting potential of the IOL [25], and uncorrected or inappropriate correction of astigmatism results in decreased vision [26].

Our evaluation of pre- and postoperative data collected during the first postoperative year after the implantation in 35 eyes (of 22 astigmatic cataract patients) of the Bi-Flex 677TA(Y) monofocal, toric IOL revealed that this lens is safe and highly efficient in correcting both spherical and cylindrical errors. Excellent UDVA was measured at each follow-up visit (this study shows data from the 3-month and 12-month follow-up visits only). Mean UDVA at 12 months was $0.93 \pm 0.13$ (decimal). This is comparable to the results published by Bachernegg et al. [15] for the same lens (UDVA $=0.06 \pm 0.16$; $\log M A R$ ), and is even superior to the results published about other
Nováček/Němcová/Tyx/Lahodová/ Rejmont/Rozsíval/Studený 


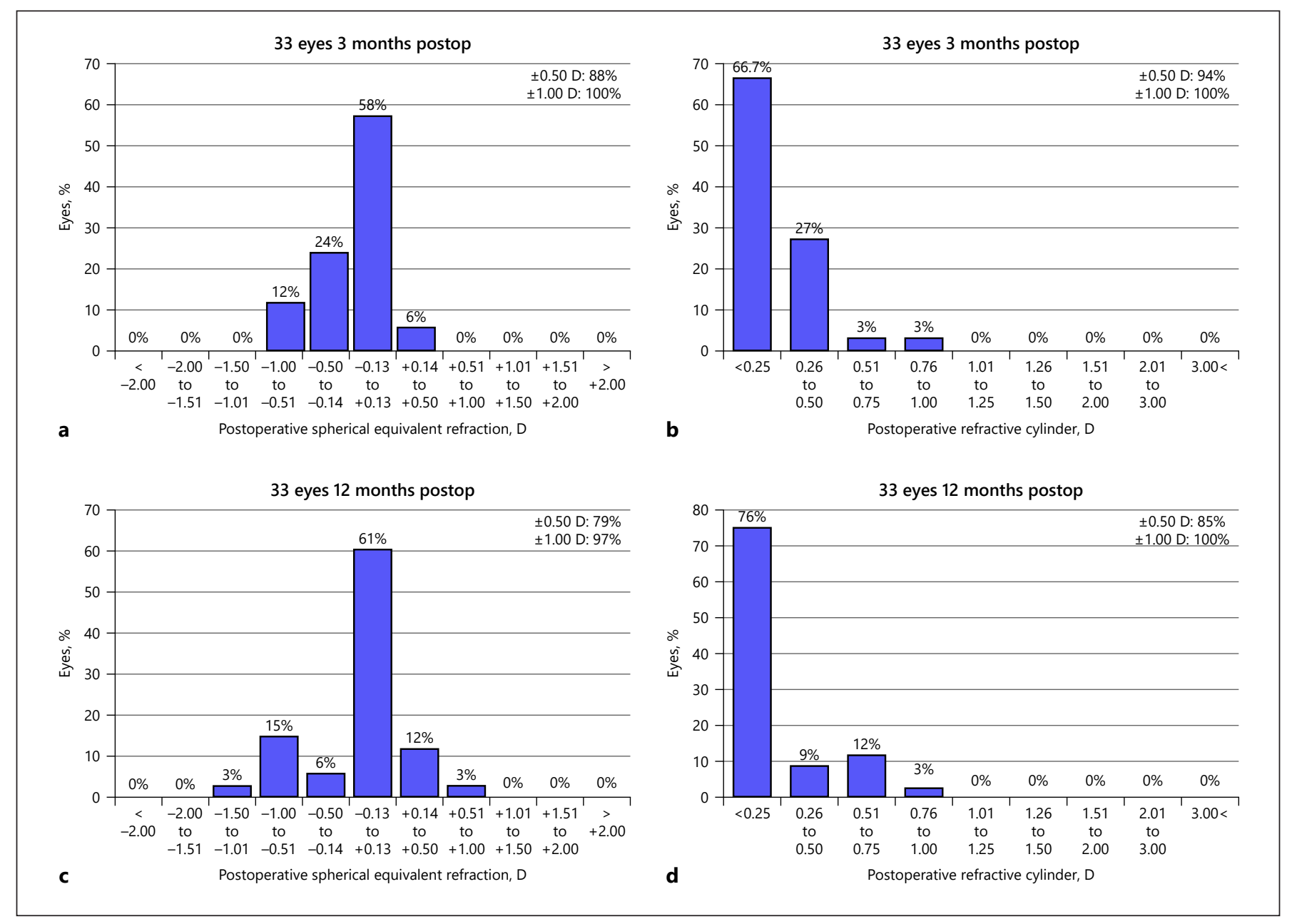

Fig. 4. Postoperative spherical equivalent refractions (SEQ) and residual cylindrical refractions (CYL) show stability over time. Results are close to the target refraction, emmetropia. a SEQ 3 months postop. b CYL 3 months postop. c SEQ 12 months postop. d CYL 12 months postop.

Fig. 5. Cumulative histogram of the magnitudes of preoperative corneal astigmatism and refractive astigmatism measured 12 months postoperatively at the corneal plane shows efficient astigmatism correction achieved by the Bi-Flex 677TA(Y) IOL after cataract surgery.

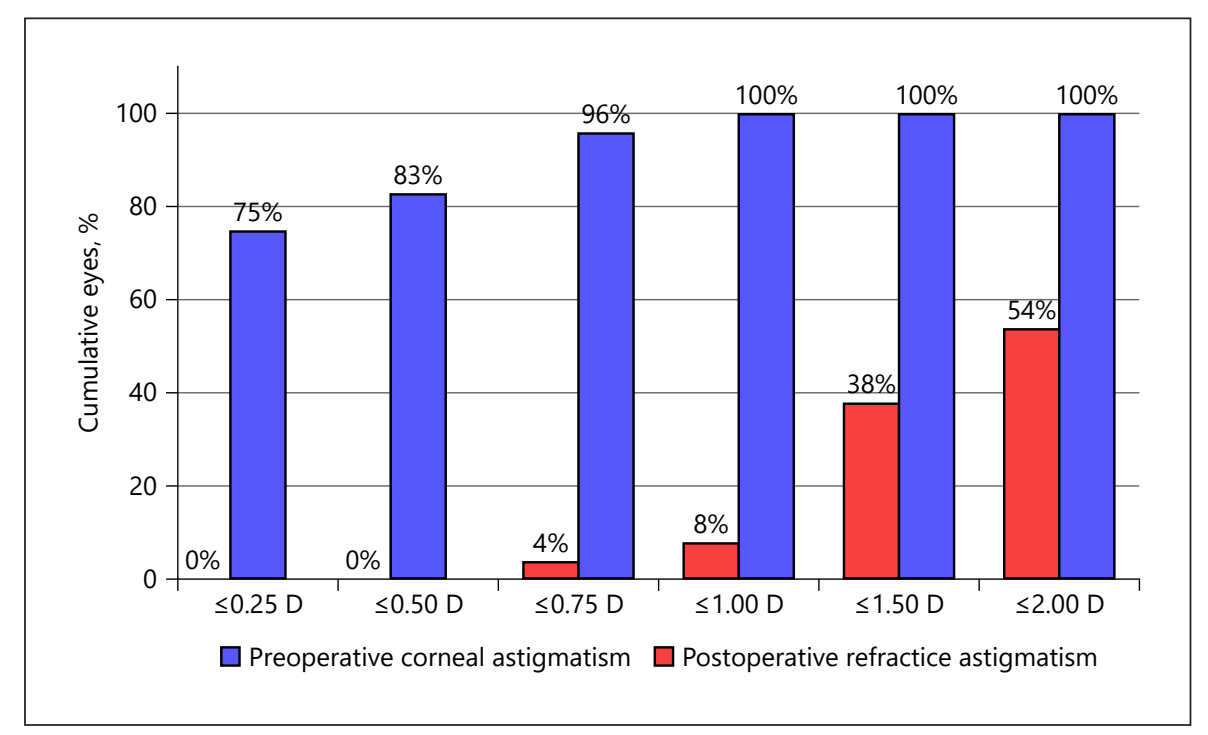




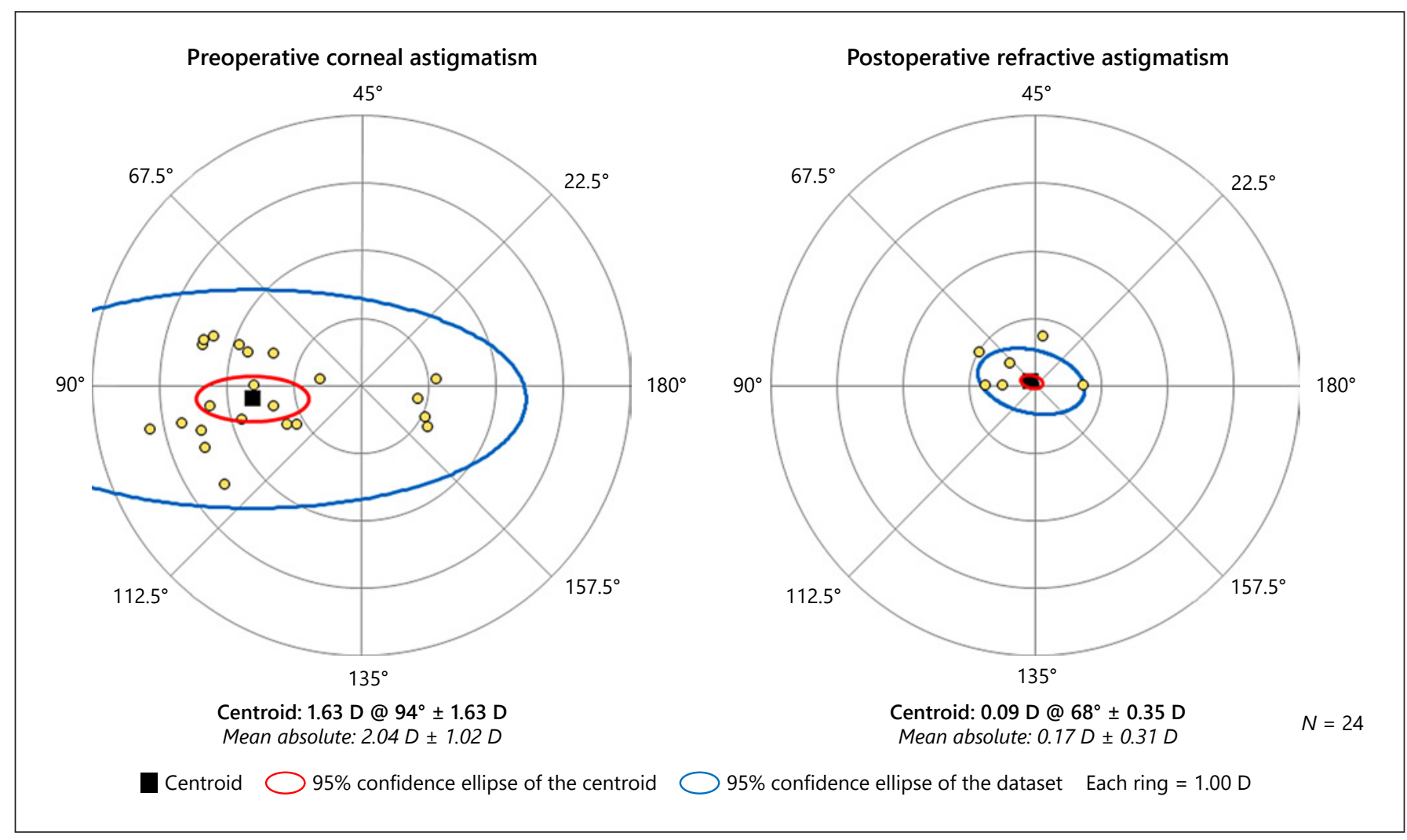

Fig. 6. Double-angle plots of preoperative and refractive astigmatism measured 12 months postoperatively. Centroid values with SDs and 95\% confidence ellipses of the dataset and of the centroid values reflect efficient astigmatism correction achieved by the Bi-Flex 677TA(Y) IOL.

toric IOLs on the market. Alió et al. [13] reported a mean UDVA of $0.65 \pm 0.22$ (decimal) achieved by the Zeiss Acri. Comfort 646TLC lens 3 months postoperatively, which is inferior to the results in our cohort. Similarly, Kawahara and Takayanagi [12] reported $0.17 \pm 0.13$ (logMAR) for the Alcon AcrySof IQ toric lens, and Jung et al. [11] found $0.09 \pm 0.09$ ( $\log$ MAR) for the Precizon toric IOL and $0.08 \pm 0.12$ (logMAR) for the Tecnis Toric lens. The visual acuity achieved with multifocal toric lenses is similar, or even somewhat lower than the results mentioned above $[27,28]$.

The Bi-Flex 677TA(Y) lens was proved to efficiently correct both spherical and cylindrical refractive errors, also in the long term. Our analysis of 33 eyes resulted in a $-0.17 \pm 0.13 \mathrm{D}$ of residual cylindrical refraction. This is much closer to the target refraction, emmetropia, than the $0.73 \pm 0.55 \mathrm{D}$ residual cylinder reported for the AcrySof IQ toric IOL [12] and the $-0.31 \pm 0.29 \mathrm{D}$ and -0.41 $\pm 0.33 \mathrm{D}$ postoperative cylinders for the Precizon and Tecnis toric IOLs, respectively [11]. Our audit shows even lower residual astigmatism than the study by Bachernegg et al. [15] did for the same IOL (postoperative CYL $=0.28$ $\pm 0.61 \mathrm{D})$. We are aware that it is not only the IOL itself, but also the patient population, the measurement techniques, the precision of the IOL calculation formulae, and the accuracy of the IOL alignment during surgery all contribute to the final refractive and visual outcomes [4-6]. However, the vector analysis we performed also confirmed that the Bi-Flex 677TA(Y) toric lens has high potential for efficiently correcting preoperative astigmatism. The 2 basic vectors, TIA and SIA, were similar to each other, which means that the cylindrical refractions that were actually achieved were close to the intended target. These 2 vectors determined the DV, which was consequently low $\left(\mathrm{DV}=0.17 ; 0.09 \mathrm{Ax} 158^{\circ}\right)$, pointing to efficient astigmatism correction. This result is in agreement with the high CI of 0.96 , which is close to the optimal value of 1.00; however, it also reflects a slight undercorrection of astigmatism. If we compare these results to the corresponding values published for other toric IOLs, we 


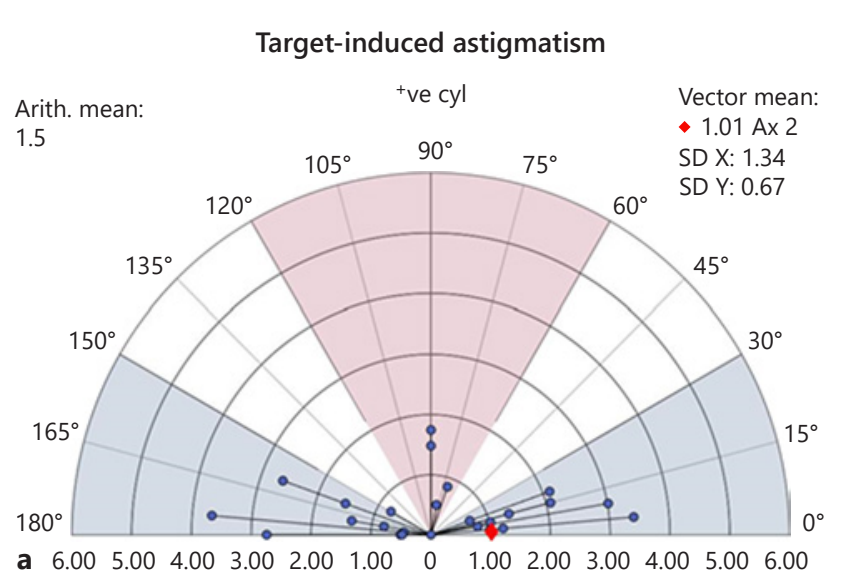

Difference vector

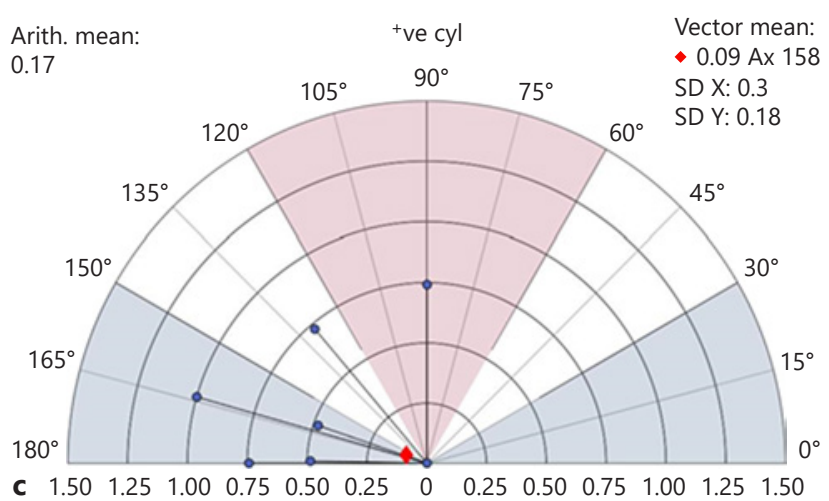

Surgically induced astigmatism

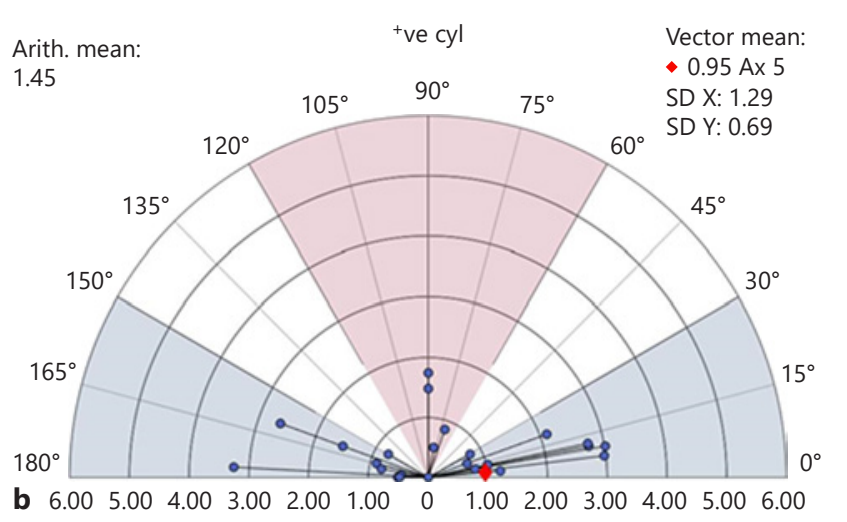

Correction index

Arith. mean:

$0.96 \quad 105^{\circ} \quad 90^{\circ} \quad 75^{\circ}$

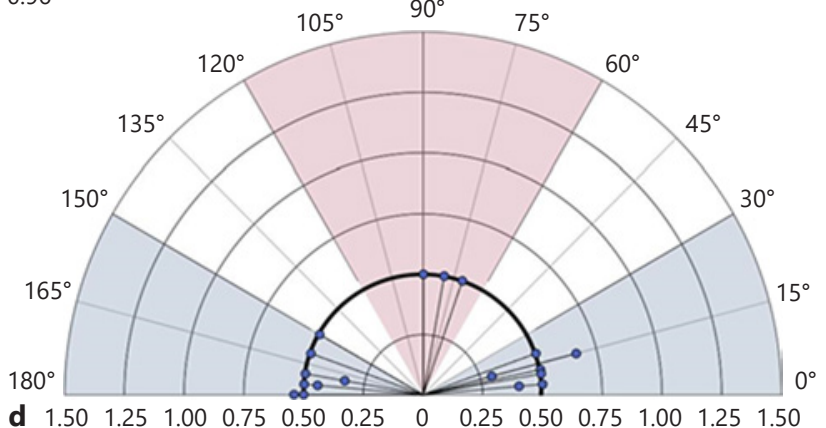

Fig. 7. a Target-induced astigmatism. b Surgically induced astigmatism. $\mathbf{c}$ The difference vector. $\mathbf{d}$ The correction index based on preoperative corneal and postoperative manifest refractions measured 12 months after toric IOL implantation. Calculations were performed by the Alpins vector analysis method.

can conclude that the monofocal, toric Bi-Flex 677TA(Y) lens is a good option for cataract patients with preoperative corneal astigmatism (Table 4).

During the first postoperative year following implantation of the Bi-Flex 677TA(Y) lenses, no off-axis rotation of $>5^{\circ}$ could be observed (Table 4 ). In contrast, in a recent study, Piovella et al. [28] reported that only $93.8 \%$ of the eyes implanted with the AT LISA tri $939 \mathrm{MP}$ toric lens had a rotation of $\leq 5^{\circ} 1$ year after surgery. The superior rotational stability of the Bi-Flex lens is ensured by its special double-loop haptics, which have a large contact angle (2 $\times 88.8^{\circ}$ ) with the wall of the capsular bag [19]. This comparison with the AT LISA tri 939MP toric lens also confirms the hypothesis that plate haptic IOLs may be less stable in the long term, especially in large eyes [9].

Astigmatism Correction with a Double-

Loop Haptic IOL
Taking into consideration that no severe adverse events or complications were observed during the first postoperative year after the IOL implantation, and based on a detailed analysis of the study population, we conclude that the Bi-Flex 677TA(Y) toric IOL is a reliable and efficient solution for cataract patients who also wish to receive treatment for their preoperative corneal astigmatism.

Our intention is to perform a prospective study on the Liberty 677 MTY trifocal toric IOL by the same manufacturer, as the Liberty IOL has the same stable Bi-flex design, and astigmatism correction is widely known to be a key factor in achieving the most favorable visual outcome with multifocal lenses, and consequently providing patients independence from wearing spectacles and an uncompromised quality of vision. 


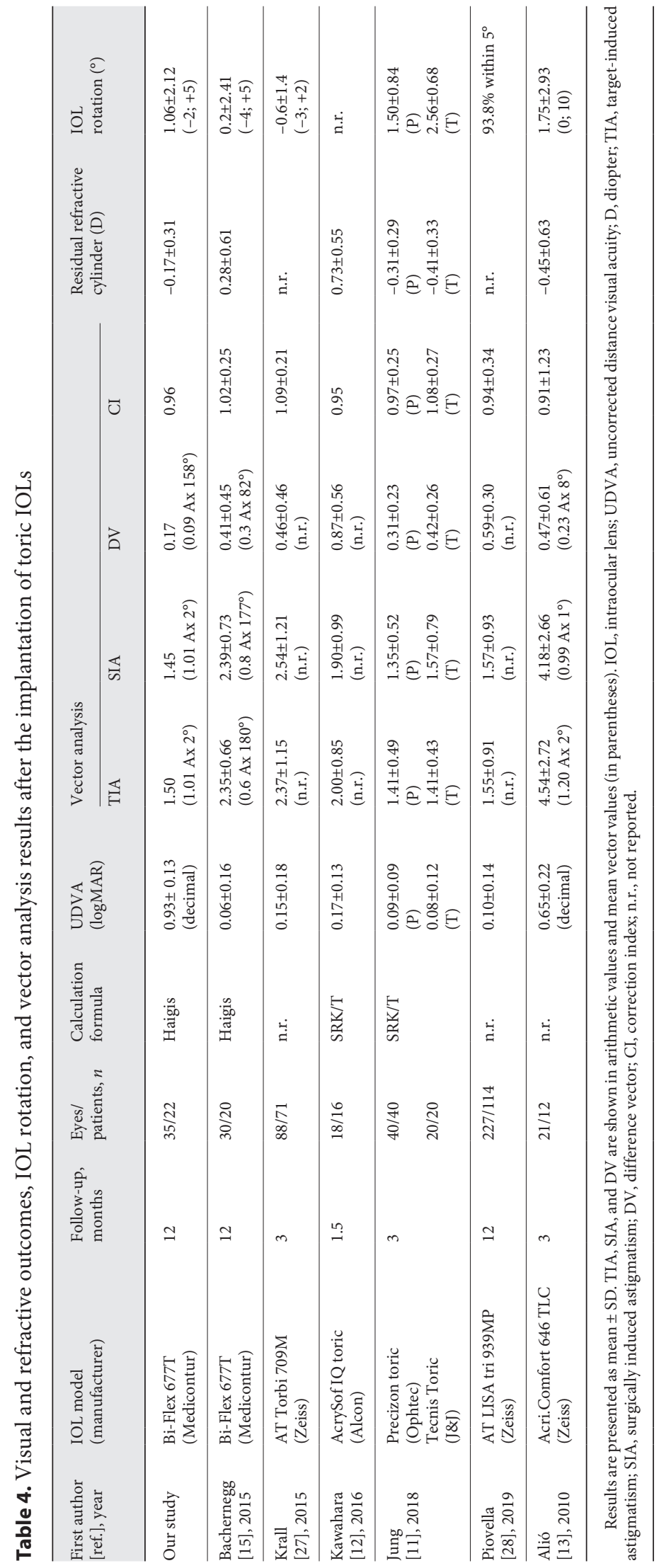

\section{Statement of Ethics}

As the data were obtained retrospectively, according to Institutional Review Board and national regulations, no ethics approval was required for the current clinical evaluation. The data management and the entire clinical study adhered to the principles of the Declaration of Helsinki. Each patient gave their informed consent to participate.

\section{Conflict of Interest Statement}

The authors have no conflicts of interest to declare.

\section{Funding Sources}

There was no funding.

\section{Author Contributions}

L.V.N. was the general designer, main coordinator, and surgeon of this study. He took part in drafting the manuscript. M.N. was responsible for calculation of the power of the implanted IOLs, examination of patients before surgery, follow-up, registration of IOL-axis, acquisition of data from all examination work, and assessment of postoperative results. K.T. performed the Pentacam, endothelial microscopy, OCT, and HD analyzer examinations, collected data and completed the protocols. K.L. was responsible for preparing patients in the operating room, assistance during surgery, control of perioperative procedures (administration of medication, etc.), completing perioperative forms, and recording undesirable events. L.R. was responsible for examining all retinal findings, solving any vitreoretinal complications in cases needing vitreoretinal surgery, and critical assessment of retinal findings. P.R. was the general advisor of the project. He assessed the material of the IOL and the surgical protocol and helped in data interpretation. He also performed critical revision of the work. P.S. advised us on the choice of a suitable operative technique, helped with the interpretation of corneal findings pre- and postoperatively, and supervised the entire project.

References
1 Nagy ZZ, McAlinden C. Femtosecond laser cataract surgery. Eye Vis (Lond). 2015 Jun; 2(1):11.

2 Ferrer-Blasco T, Montés-Micó R, Peixoto-deMatos SC, González-Méijome JM, Cerviño A. Prevalence of corneal astigmatism before cataract surgery. J Cataract Refract Surg. 2009 Jan;35(1):70-5

3 Ferreira TB, Hoffer KJ, Ribeiro F, Ribeiro P, O'Neill JG. Ocular biometric measurements in cataract surgery candidates in Portugal. PLoS One. 2017 Oct;12(10):e0184837. 
4 Abulafia A, Hill WE, Franchina M, Barrett GD. Comparison of methods to predict residual astigmatism after intraocular lens implantation. J Refract Surg. 2015 Oct;31(10):699707.

5 Abulafia A, Koch DD, Wang L, Hill WE, Assia EI, Franchina M, et al. New regression formula for toric intraocular lens calculations. J Cataract Refract Surg. 2016 May;42(5):66371.

6 Titiyal JS, Kaur M, Jose CP, Falera R, Kinkar A, Bageshwar LM. Comparative evaluation of toric intraocular lens alignment and visual quality with image-guided surgery and conventional three-step manual marking. Clin Ophthalmol. 2018 Apr;12:747-53.

7 Ferreira TB, Berendschot TT, Ribeiro FJ. Clinical outcomes after cataract surgery with a new transitional toric intraocular lens. J Refract Surg. 2016 Jul;32(7):452-9.

8 Bascaran L, Mendicute J, Macias-Murelaga B, Arbelaitz N, Martinez-Soroa I. Efficacy and stability of AT TORBI 709 M toric IOL. J Refract Surg. 2013 Mar;29(3):194-9.

9 Seth SA, Bansal RK, Ichhpujani P, Seth NG. Comparative evaluation of two toric intraocular lenses for correcting astigmatism in patients undergoing phacoemulsification. Indian J Ophthalmol. 2018 Oct;66(10):1423-8.

10 Alberdi T, Macías-Murelaga B, Bascarán L, Goñi N, de Arregui SS, Mendicute J. Rotational stability and visual quality in eyes with Rayner toric intraocular lens implantation. J Refract Surg. 2012 Oct;28(10):696-701.

11 Jung NY, Lim DH, Hwang SS, Hyun J, Chung TY. Comparison of clinical outcomes of toric intraocular lens, Precizon vs Tecnis: a single center randomized controlled trial. BMC Ophthalmol. 2018 Nov;18(1):292.
12 Kawahara A, Takayanagi Y. Vector analysis investigation of toric intraocular lens with no deviation from the intended axis. Clin Ophthalmol. 2016 Nov; 10:2199-203.

13 Alió JL, Agdeppa MC, Pongo VC, El Kady B. Microincision cataract surgery with toric intraocular lens implantation for correcting moderate and high astigmatism: pilot study. J Cataract Refract Surg. 2010 Jan;36(1):44-52.

14 Bachernegg A, Rückl T, Riha W, Grabner G, Dexl AK. Rotational stability and visual outcome after implantation of a new toric intraocular lens for the correction of corneal astigmatism during cataract surgery. J Cataract Refract Surg. 2013 Sep;39(9):1390-8.

15 Bachernegg A, Rückl T, Strohmaier C, Jell G, Grabner G, Dexl AK. Vector analysis, rotational stability, and visual outcome after implantation of a new aspheric toric IOL. J Refract Surg. 2015 Aug;31(8):513-20.

16 World Medical Association. World Medical Association Declaration of Helsinki: ethical principles for medical research involving human subjects. JAMA. 2013 Nov;310(20): 2191-4.

17 Alpins NA. A new method of analyzing vectors for changes in astigmatism. J Cataract Refract Surg. 1993 Jul;19(4):524-33.

18 Alpins N. Astigmatism analysis by the Alpins method. J Cataract Refract Surg. 2001 Jan; 27(1):31-49.

19 Contact angle measurements of the lens measured according to the method described in ISO 11979-3, compressed to $9.0 \mathrm{~mm}$ overall diameter. Courtesy of the Research and Development Department of Medicontur Medical Engineering Ltd (2019).

20 Medicontur IOL Optimizer [Internet]. Available from: https://toriccalculator.net.
21 Haigis W. IOL calculation according to Haigis. Available from: http://www.augenklinik. uniwuerzburg.de/uslab/ioltxt/haie.htm, last revision: 7 December 1998.

22 Abulafia A, Koch DD, Holladay JT, Wang L, Hill W. Pursuing perfection in intraocular lens calculations: IV. Rethinking astigmatism analysis for intraocular lens-based surgery: Suggested terminology, analysis, and standards for outcome reports. J Cataract Refract Surg. 2018 Oct;44(10):1169-74.

23 Novacek L. Evaluation of refractive and visual outcomes, as well as astigmatism-correcting efficiency and rotational stability of a double-loop haptic toric intraocular lens - a oneyear follow-up, Mendeley Data. 2020, Online ahead of print.

24 Reinstein DZ, Archer TJ, Srinivasan S, Mamalis N, Kohnen T, Dupps WJ Jr, et al. Standard for Reporting Refractive Outcomes of Intraocular Lens-Based Refractive Surgery. J Refract Surg. 2017 Apr;33(4):218-22.

25 Roach L, Berdahl JP, Chu YR, Hill WE. Toric IOLs: Four Options for Addressing Residual Astigmatism. EyeNet Magazine (American Academy of Ophthalmology) 2012 April. Available from: https://www.aao.org/eyenet/ article/toric-iols-four-options-addressingresidual-astigm.

26 Wolffsohn JS, Bhogal G, Shah S. Effect of uncorrected astigmatism on vision. J Cataract Refract Surg. 2011 Mar;37(3):454-60.

27 Krall EM, Arlt EM, Hohensinn M, Moussa S, Jell G, Alió JL, et al. Vector analysis of astigmatism correction after toric intraocular lens implantation. J Cataract Refract Surg. 2015 Apr;41(4):790-9.

28 Piovella M, Colonval S, Kapp A, Reiter J, Van Cauwenberge F, Alfonso J. Patient outcomes following implantation with a trifocal toric IOL: twelve-month prospective multicentre study. Eye (Lond). 2019 Jan;33(1):144-53. 